\title{
The effect of pulsatile blood flow on blood pressure regulatory mechanisms
}

\author{
John V. Ringwood ${ }^{1}$, Federica Taussi ${ }^{2}$ and Annraoi M. de Paor ${ }^{3}$
}

\begin{abstract}
This paper demonstrates that the effect of pulsatile blood flow on the baroreflex is to effectively reduce the gain of the baroreflex loop. This has important implications for both the development of integrative physiology models, which do not include pulsatile blood flow, and the use of non-pulsatile ventricular assist devices with either replace the heart or assist the heart in achieving adequate blood circulation. To elucidate the effect of the pulsatile nature of blood flow, we utilise the concept of an equivalent nonlinearity to replace the baroreflex curve, driven by a pulsatile blood flow/pressure signal, with an equivalent nonlinearity corresponding to a non-pulsatile situation. Tests are performed on a feedback model for the peripheral resistance baroreflex and conclusions made to the stability implications, using a describing function analysis.
\end{abstract}

\section{INTRODUCTION}

The literature contains a vast array of mathematical models for the circulatory system, at various levels of detail and focussing on a greater or lesser part of the complete circulatory system, depending on the objective. In some of these models, mostly those considering a fine level of time resolution, the pulsitility of blood flow is modelled. However, in the main, pulsitility is circumvented, either by considering physiological quantities such as blood flow $(\mathrm{BF})$, or blood pressure (BP), on an averaged basis (eg. mean arterial flow, pressure) or by modelling $\mathrm{BP}$ or $\mathrm{BF}$ on a beat-to-beat basis. While a discrete-time (or, rather, discrete event) beat-to-beat analysis avoids the need for interpolation between measurement points, it precludes the use of frequency-domain analysis tools based on regularly sampled time signals. The averaging approach, for a great number of applications, can be justified on the basis of the filtering effect of arterial compliance. However, we also note that some components involved in circulatory control, viz. the baroreceptors, are especially sensitive to relatively high frequencies, including the pulsatile frequency of the heart [1], [2].

For example, the well-known integrative model of Guyton [3] ignores pulsitility, though heart-rate is, of course, considered in terms of its impact on cardiac output. The focus in Guyton's model is the simulation of overall homeostasis and longer-term transient effects, rather than sub-second analysis. In contrast, the model of Monti et al [4] focusses on short time scales and models each heart chamber, though

\footnotetext{
${ }^{1}$ J.V. Ringwood is with the Dept. of Electronic Engineering at the National University of Ireland, Maynooth, County Kildare, Ireland.

${ }^{2} \mathrm{~F}$. Taussi is with CME Societ Cooperativa Consortile, Via V. Nenni 16, 60127 Ancona, Italy.

${ }^{3}$ A.M. de Paor is Emeritus Professor with the School of Electrical, Electronic and Communications Engineering, National University of Ireland, Dublin, Belfield, Dublin, Ireland.
}

cardiovascular control is subsequently considered on a beatto-beat basis.

Our contention is that, whether blood flow pulsitility is explicitly modelled or not, the effect of pulsitility must be accounted for. In particular, it has been shown [5], via simulation analysis, that varying levels of blood pressure pulsitility can have a significant effect on baroreflex gain, a phenomenon which is confirmed by our analysis in this paper. While it may usually be the case that baroreflex gains and response curves are measured under pulsatile conditions, the use of such parametric descriptions in non-pulsatile and pulsatile models needs to be carefully monitored. For example, if the gains around a baroreflex loop are experimentally measured and subsequently modelled, care must be taken not to further include the effects of pulsitility in the model.

Another area where the gain effects of pulsitility are important is the area of artificial hearts or left ventricular assist devices (LVADs). Typically, blood flow in an artificial heart is provided by an axial turbine, resulting in an absence of pulsitility in blood flow and pressure, though some devices (e.g. the AbioCor artificial heart) have a pumping action. However, non-pulsatile artificial hearts generally have advantages of greater durability and smaller size than their pulsatile counterparts. Where a non-pulsatile LVAD is used to supplement the output of an underperforming heart, some residual pulsitility may be retained. It is apparent that it is easier for nature to produce a pulsatile pump, but whether pulsitility is a requirement for a number of important physiological regulatory mechanisms remains a moot point, though some studies have examined the relative effects of pulsatile and non-pulsatile devices in animals [6], [7]. However, the true long term effects of a pulseless circulatory system have yet to be understood, though some progress has been made [8].

This paper focuses on the impact of pulsitility on a specific section of the circulatory system: the peripheral resistance baroreflex. In particular, we develop an analytical technique which can articulate how pulsatile blood flow can modulate baroreflex gain. While previous simulation analysis has shown there to be a modulating effect [5], to the best of the authors' knowledge no analytical tools currently exist to examine such an effect. We would contend that, while simulation can give some insight into phenomena which occur under particular scenarios, analytical (and, in particular, algebraic) tools have a greater capability to give the global picture and show the underlying characteristics which generate the phenomenon. Some further evidence of the apparent change in baroreflex gain can be ascertained from the 
relative increase in Meyer wave [9] activity following LVAD insertion [10]. However, the authors in [10] use the Meyer wave activity to support their contention that low frequency (LF) oscillations emanate from a central source, while we offer the increased activity as solid evidence of increased baroreflex gain due to a reduction in pulsitility, following the limit-cycle oscillation explanation for LF oscillations [11]. We also concede, of course that, following LVAD insertion, baroreflex gain in the peripheral resistance loop may also change due to higher average blood pressure and flow.

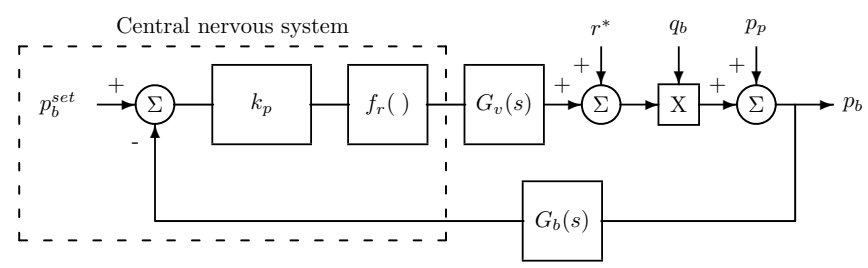

Fig. 1. Essential baroreflex components

\section{Model OF THE BAROREFLEX}

Fig.1 shows the essential components of the baroreflex which relate to the neural control of blood pressure, including the dynamical system components (including delays), nonlinearities, and blood flow pulsitility. In this study, we will concentrate solely on the sympathetic control of peripheral resistance and assume that blood flow is relatively constant, aside from the pulsatile component. We appreciate that this is a significant approximation, but it is an important first step in establishing the fundamental effect of pulsitility on the neural baroreflex. We ignore arterial compliance in this preliminary study for the simple reason that we model the pulsatile signal in the ascending aorta, which is the region in which blood pressure is measured via the baroreceptors (aortic arch).

The central nervous system (CNS) is assumed to contain a notional blood pressure set-point, $p_{b}^{\text {set }}$ while $f_{r}()$ describes the static (steady-state) characteristics relating blood pressure to sympathetic resistance nerve activity, via the generic arctan function description:

$$
y=f_{r}(x)=h \tan ^{-1}\left(\beta\left(x-x^{*}\right)\right)+y *
$$

Note that the parameterisation of the static baroreflex characteristic by an arctan function allows for a relatively simple describing function to be calculated, facilitating straightforward stability analysis [12]. A set of parameters for the arctan function for a rabbit in the normoxia case [12] is specified in Table I, with a typical fit to experimental data shown in Fig.2.

TABLE I

ARCTAN PARAMETER VALUES FOR NORMOXIA CASE

\begin{tabular}{|c|c|c|c|}
\hline$h$ & $\beta$ & $x^{*}$ & $y^{*}$ \\
\hline 33 & 0.12 & -71 & 55 \\
\hline
\end{tabular}

The dynamic components of the model in Fig.1 are:

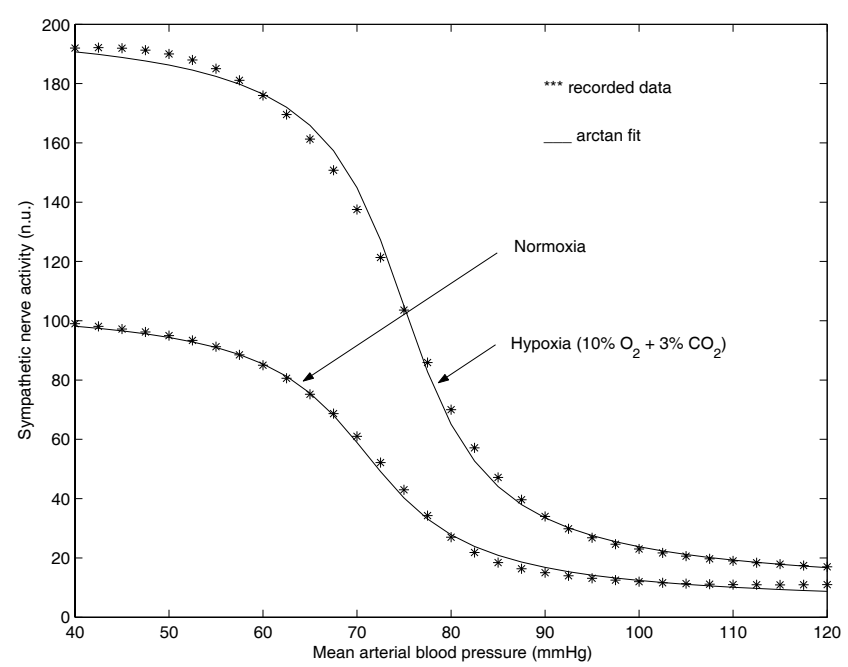

Fig. 2. Arctan fit for experimental data [12]

$$
G_{v}(s)=\frac{e^{-s \tau_{e}}}{1+s \tau_{v}} \quad, \quad G_{b}(s)=e^{-s \tau_{a}}
$$

Note that:

- $\tau_{a}$ represents afferent (both pre- and post-ganglionic) nerve delay,

- $\tau_{e}$ represents efferent nerve delay,

- The dynamic lag of the vasculature, $\tau_{v}$, is primarily due to the dynamics of contraction of the smooth muscle surrounding the arterioles, and

- For convenience, the gain term, $k_{p}$, will be absorbed into the input scaling term, $\beta$, of the arctan function as:

$$
\beta^{*}=\beta k_{p}
$$

Note also that the steady-state (dc) gain of $G_{v}(s)$ has been normalised. This is partly due to the fact that there is some ambiguity over the dc gain between efferent sympathetic nerve activity (SNA) and mean arterial pressure (MAP) and partly due to the fact that such gain has been absorbed elsewhere (in $k_{p}$ and $f_{r}()$ ). The model, via the values in Table II, is parameterised for the rabbit [13] and we note that the model parameters are species dependent [11].

TABLE II

MODEL PARAMETER VALUES

\begin{tabular}{|c|c|c|c|}
\hline$k_{p}$ & $\tau_{e}$ & $\tau_{a}$ & $\tau_{v}$ \\
\hline 3.0 & 0.67 & 0.2 & 10.0 \\
\hline
\end{tabular}

We complete the definition of the quantities in Fig.1 with:

$r^{*} \quad$ is the baseline peripheral resistance not resulting from neural influences,

$q_{b} \quad$ is blood flow, or cardiac output, and

$p_{p} \quad$ is the pulsatile component of the blood pressure signal. 


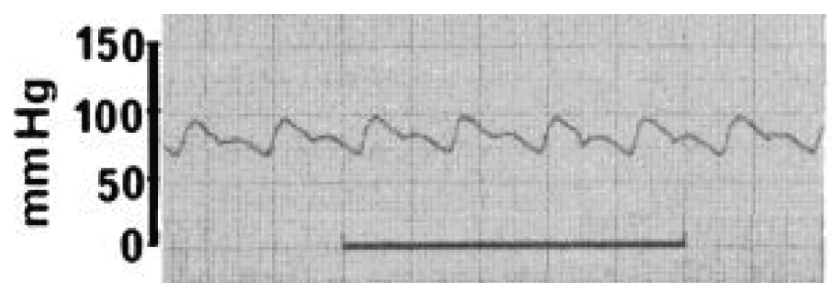

Fig. 3. Blood pressure measurement in the ascending aorta of a rabbit

\begin{tabular}{|c|c|c|c|c|c|}
\hline$T(\mathrm{~s})$ & $t_{1}(\mathrm{~s})$ & $t_{2}(\mathrm{~s})$ & $t_{3}(\mathrm{~s})$ & $A_{1}(\mathrm{mmHg})$ & $A_{2}(\mathrm{mmHg})$ \\
\hline 0.25 & 0.09 & 0.03 & 0.13 & 20 & 40 \\
\hline
\end{tabular}

TABLE III

PARAMETERS FOR PULSATILE DITHER SIGNAL

\section{A. The pulsatile blood signal}

Here, we will specify the signal which describes the periodic oscillations in blood pressure due to heart pulsatility. Although blood pulsatility originally derives from flow variations, it is convenient for us to use a pressure representation. Avolio et al [14] describes the blood pressure waveform for a rabbit (the time calibration indicated by the dark horizontel line corresponds to $1 \mathrm{~s}$ ), shown in Fig.3 which we will approximate using the piecewise constant function shown in Fig.4. While the sharp corner at the diastole is somewhat more acute that the signal measured by Avolio et al, this approximation leads to a more simplified analysis and we will show later that the effect of pulsatility on the baroreflex is not so sensitive to small details in the shape of the pulsatile pressure variations.

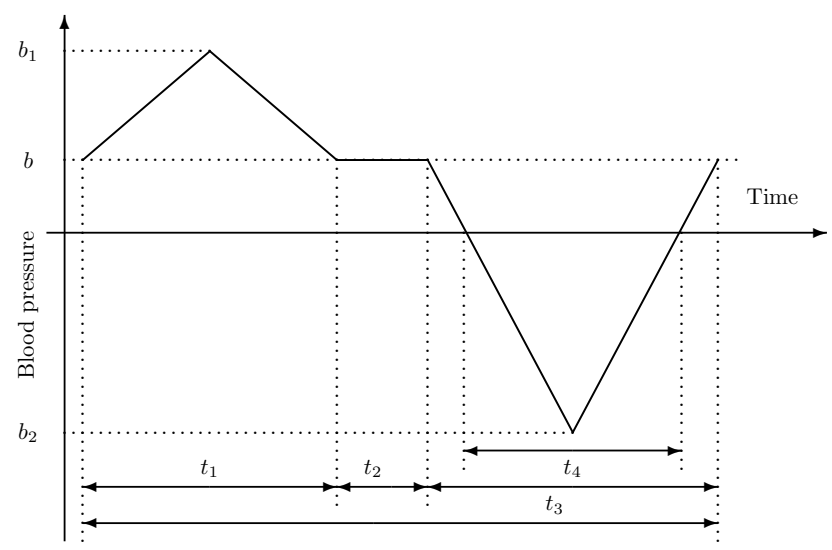

Fig. 4. Piecewise linear approximation of blood pressure signal

In the forthcoming analysis, we will represent the blood pressure signal by its slowly varying value $r$ plus a zero mean 'dither' signal, $d(t)$, represented by the signal in Fig.4. The measured parameters of the pulsatile pressure signal are shown in Table III.

Note that, initially, $b$ is specified as a free parameter and then determined, using simple geometry, to ensure that the dither signal has zero mean, resulting in $b=6.8 \mathrm{mmHg}$. We

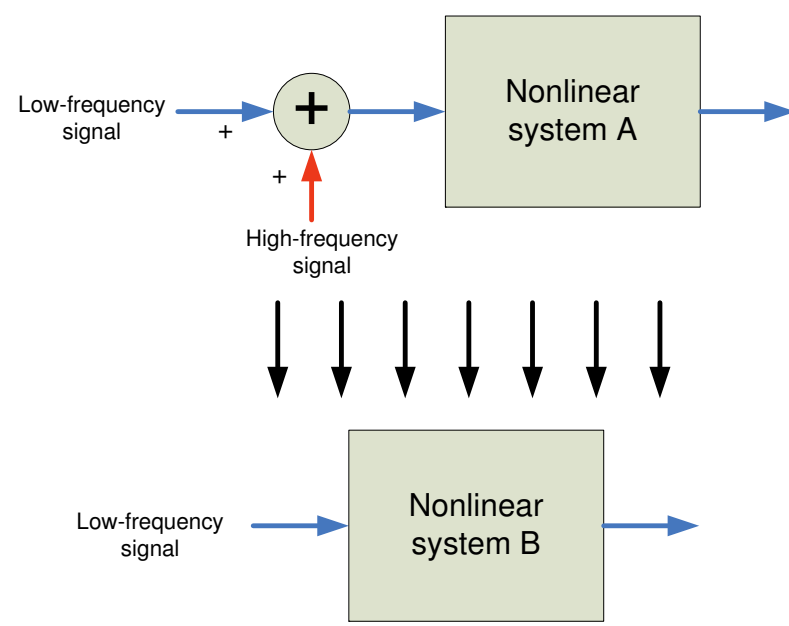

Fig. 5. Equivalent non-linearity concept

also define:

$$
A_{1}=b_{1}-b \quad, \quad A_{2}=b_{2}+b
$$

\section{EQUIVALENT NON-LINEARITY}

The concept of an equivalent non-linearity for a nonlinear characteristic subject to a (relatively) high frequency dither signal has been traced back to J.C. Lozier of Bell Labs in 1950 [15], [16]. It can be used both as an analysis technique to examine the effect of a combination of a high frequency dither signal and a non-linearity, or as a synthesis technique, where a dither signal is specially constructed and injected into a system in order to produce a more desirable (probably less severe) non-linear function. It is the former case that is addressed in this paper.

\section{A. Equivalent non-linearity concept}

The equivalent nonlinearity concept addresses the system as shown in Fig.5. The original non-linear block is specified by $y=f(u)$, while the equivalent non-linearity of $f()$ with the addition of the dither signal is specified by $\bar{y}=g(r)$. We note that the equivalent nonlinearity method requires that the dither signal appear at the input to the nonlinearity. To this end, we can easily move the pulsatile component of blood pressure, $p_{p}$, in Fig. 1 to the input of $f_{r}()$, with the following observations:

- The effect of $G_{b}(s)$ is simply a pure delay, resulting in a phase shift in the pulsatile signal, which has no effect on the equivalent nonlinearity calculation,

- The effect of the summation junction results in just a sign change, and

- $k$, representing the central nervous system 'gain' has a scaling effect on the dither signal.

It can be shown that the equivalent non-linearity of the single-valued function $y(t)=f(u(t))$, with $u=r+d(t)$, where $r$ is a (relatively) low frequency signal and $d(t)$ a 
(relatively) high frequency dither signal, is given by $g(r)$, where:

$$
\bar{y}=g(r)=\int_{-\infty}^{\infty} f(u) p(u-r) d u
$$

where $p()$ is a weighting function related to a probability density function on $d(t)$. The technique relies on two assumptions:

- The frequency of the dither waveform, $d(t)$, lies above the bandwidth of the dynamic system, $G_{v}(s)$, which follows the non-linear element, and

- The spectral difference between $r(t)$ and $d(t)$ is such that, over a dither period, $T$, no significant error is incurred by regarding $r(t)=r$ as constant.

\section{B. Equivalent non-linearity calculation method}

In general, for a single-valued non-linearity, $f()$, with input $u(t)=r+d(t)$, with $d(t)$ being the dither signal [16], and $r$ being relatively constant over the dither period,

$$
y=f(r+d(t))
$$

Let $p(q) d q$ be the probability that, for any time $t$, chosen at random, $d(t)$ lies in the range $q$ to $q+d q$, with $p(q)$ being the probability density function for the dither. When $d(t)$ has the value $q$,

$$
y=f(r+q)
$$

the expected value for $y$ is:

$$
\bar{y}=\int_{-\infty}^{\infty} f(r+q) p(q) d q
$$

To calculate $p(q)$, let $F(q)$ be the probability that $d(t)$ lies above $q$,

$$
F(q)=\int_{q}^{\infty} p(q) d q=-\int_{\infty}^{q} p(q) d q
$$

and $p(q)$ can now be determined from:

$$
\frac{d F(q)}{d q}=-p(q)
$$

\section{Equivalent nonlinearity calculation}

Since the pulsatile signal has three distinct component parts, with durations $t_{1}, t_{2}$ and $t_{3}$ as shown in Fig.4, the equivalent nonlinearity $\bar{y}$ can be calculated as the sum of the equivalent nonlinearities associated with each component, weighted by their time duration. The weighting factors, $\alpha_{i}$, are the portions of the total period occupied by the $i^{t h}$ pulsatile component, so that $\alpha_{i}=t_{i} / T$. Initially, we calculate the function $F(q)$, which is the probability that $d(t)$ lies above value $q$. For the first component of the dither signal in Fig.4, i.e. a triangle with offset $b$ and amplitude $A_{1}, F(q)$ is calculated as:

$$
\begin{aligned}
F(q) & =\frac{A_{1}+b-q}{2 A_{1}} \quad \text { if } b<q<A_{1}+b \\
& =0 \quad \text { if } q>A_{1}+b \\
& =1 \text { if } q<b
\end{aligned}
$$

Now, from (10),

$$
p_{1}(q)=-\frac{d F(q)}{d q}=\frac{1}{2 A_{1}}
$$

and the equivalent nonlinearity for component 1 is:

$$
\begin{aligned}
\bar{y}_{1} & =G_{1}(r)=\int_{-\infty}^{+\infty} \frac{1}{2 A_{1}}\left[h \tan ^{-1}(\beta(r+q))+y^{*}\right] d q \\
& =\int_{b-A_{1}}^{b+A_{1}} \frac{1}{2 A_{1}} h \tan ^{-1}(\beta(r+q)) d q \\
& +\int_{b-A_{1}}^{b+A_{1}} \frac{1}{2 A_{1}} y^{*} d q \\
& =\frac{h}{2 A_{1} \beta} \int_{b-A_{1}}^{b+A_{1}} \beta \tan ^{-1}(\beta(e+q)) d q+\left.\frac{y^{*}}{2 A_{1}} q\right|_{b-A_{1}} ^{b+A_{1}} \\
& =\left.\frac{h}{2 A_{1} \beta}(\beta(r+q)) \tan ^{-1}(\beta(r+q))\right|_{b-A_{1}} ^{b+A_{1}} \\
& -\left.\frac{h}{4 A_{1} \beta} \ln \left(1+\beta^{2}(r+q)^{2}\right)\right|_{b-A_{1}} ^{b+A_{1}}+y^{a s t}
\end{aligned}
$$

The second dither component is a straight line with offset $b$, so the equivalent nonlinearity for this signal is simply the original nonlinearity offset by $b$. In this case, the probability function $F(q)$ is constant, with zero derivative. The equivalent nonlinearity for this component is:

$$
\bar{y}_{2}=G_{2}(r)=h \tan ^{-1}(\beta(r+b))+y^{*}
$$

The third dither component is an inverted triangle with offset $b$, which can be evaluated in a manner similar to component 1. Finally, we can determine the overall equivalent nonlinearity as:

$$
\bar{y}=G(r)=\alpha_{1} G_{1}(r)+\alpha_{2} G_{2}(r)+\alpha_{3} G_{3}(r)
$$

with $\alpha_{1}=0.36, \alpha_{2}=0.12$ and $\alpha_{3}=0.52$ and noting that:

$$
\sum_{i=1}^{3} \alpha_{i}=1
$$

\section{MAin Result}

For the set of parameters described in Section II, the original arctan function and the equivalent nonlinearity, using a pulsatile dither signal as in Fig.4, are shown in Fig.6. It is clear that there is a significant gain reduction, in the area between the saturation limits, due to the presence of the pulsatile blood flow. More importantly, the converse is true; there is a significant gain increase when pulsatility is removed. For the case considered, the gain changes by approximately a factor of 3 .

Thus, we can reasonably conclude that one of the effects of pulsatility is to moderate the gain in the neural baroreflex. 


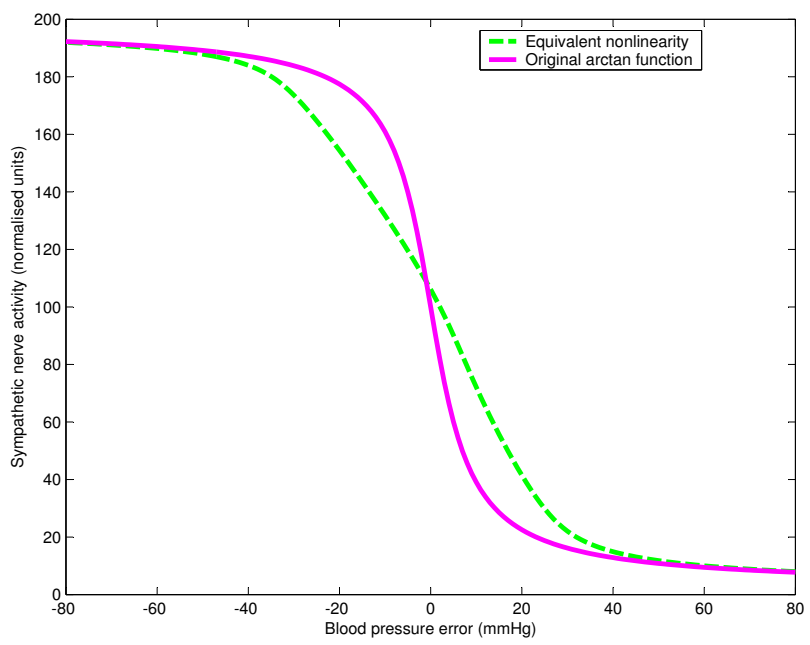

Fig. 6. Original baroreflex curve and one which includes the pulsatile effect

\section{Stability implications}

The amount of gain in the neural baroreflex has also been shown to be potentially important in the modulation of low frequency oscillations in blood pressure [11], often termed Meyer waves. A plausible theory for the generation of Meyer waves using a limit cycle analysis was proposed in [11] and the evidence of Cooley et al [10] would seem to provide further verification of this explanation, considering the clear increase in baroreflex gain in the absence of the pulsatile signal, as shown in Fig.6.

In [12], a simple describing function is developed for the arctan function, allowing some transparency between the nature/presence of potential low frequency baroreflex oscillations and the parameters of the system, including the baroreflex curve. Unfortunately, the calculation of the equivalent nonlinearity, to include the effect of pulsatility, introduces some mathematical complexity to the description of the effective nonlinearity (via (13)) which impairs the development of any simple relationships between nature/presence of potential low frequency baroreflex oscillations and the parameters of the system. However, if a reasonable (simple) approximation to the equivalent nonlinearity can be found, there is hope that the original transparency can be restored.

To this end, we propose to approximate the equivalent nonlinearity as the weighted sum of the original nonlinearity and a saturation characteristic as:

$$
N L_{\text {tot }}(r)=\gamma_{a} N L_{a}(r)+\gamma_{s} N L_{s}(r)
$$

This corresponds to a parallel combination of both nonlinearities and the resulting overall describing function is straightforwardly evaluated [17] as:

$$
D F_{\text {tot }}(M)=\gamma_{a} D F_{\text {arctan }}(M)+\gamma_{s} D F_{\text {sat }}(M)
$$

The describing function for the arctan function is given [12] as:

$$
D F_{a}(M)=\frac{2 h}{\beta M^{2}}\left(\sqrt{1+\beta^{2} M^{2}}-1\right)
$$

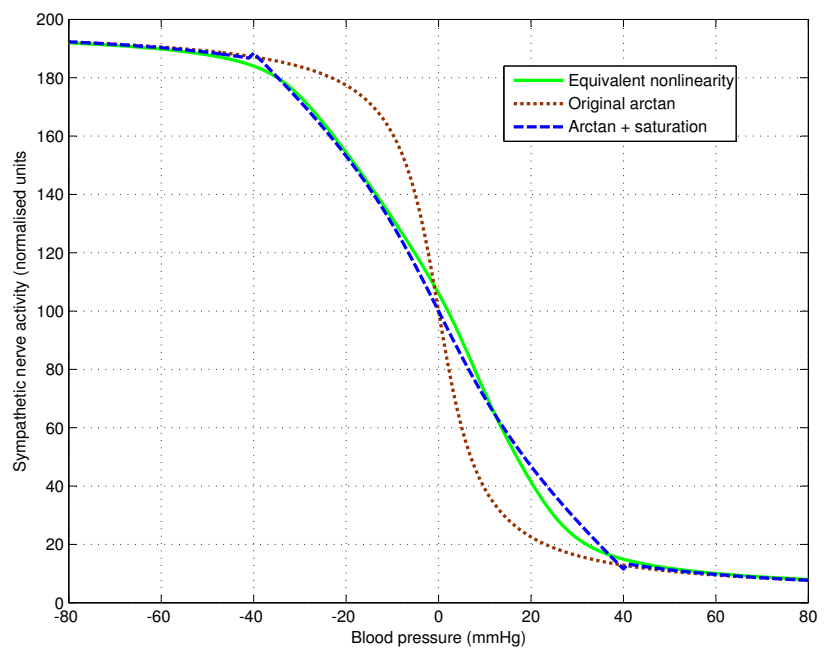

Fig. 7. Approximation to the equivalent nonlinearity

where $M$ is the amplitude of the input sinusoid, while that for a saturation characteristic [17] is:

$$
D F_{s}(M)=\left(k_{s} / \pi\right)(2 \lambda+\sin (2 \lambda))
$$

where $(-\delta, \delta)$ are the saturation limits referred to the input side, $k_{s}$ is the gain (slope) of the saturation characteristic in the linear region and $\lambda=\sin ^{-1}(\delta / M)$.

\begin{tabular}{|c|c|c|c|c|}
\hline$\beta_{\text {new }}$ & $\delta$ & $k_{s}$ & $\gamma_{a}$ & $\gamma_{s}$ \\
\hline 0.4 & 40 & 2.6 & 0.5 & 0.5 \\
\hline
\end{tabular}

TABLE IV

PARAMETERS FOR COMPOSITE APPROXIMATING NONLINEARITY

With the specification as shown in Table IV, the resulting approximation to the equivalent nonlinearity as shown in Fig.7 is achieved and the describing function for the combination is easily specified, from (16 as:

$$
\begin{aligned}
D F_{t o t}(M) & =\left(k_{s} / \pi\right)(2 \lambda+\sin (2 \lambda)) \\
& +\frac{2 h}{\beta M^{2}}\left(\sqrt{1+\beta^{2} M^{2}}-1\right)
\end{aligned}
$$

which has a simple form for the stability analysis of low frequency oscillations.

\section{CONCLUSIONS}

This paper has used the equivalent nonlinearity concept as an analytical tool to investigate the effect of pulsatility, or absence of pulsatility, in the neural baroreflex. The analysis has made some simplifying assumptions, including a focus on the peripheral resistance system, to examine the fundamental characteristics of the system with pulsatility. The main result is that there is a significant gain reduction (a factor of 3 in the case considered) which, at least, has implications for the presence/absence of low frequency oscillations in blood pressure. However, more important issues may be identified in relation to the permanently elevated baroreflex gain in LVAD patients. 
This study is preliminary, and further work is required with a more complete model of the baroreflex, to include the effects of (sympathetic and parasympathetic) heart response, a more careful consideration of arterial compliance (possibly through the use of a Windkessel model) and more authentic models of the baroreceptors and the central nervous system dynamics.

Significant differences between the shape of the blood pressure wave in different species [14], [18] would also require re-working of the equivalent nonlinearity. However, we have shown that the reduction in baroreflex gain is not so sensitive to the particular shape of the pressure wave, since the equivalent nonlinearity calculation principally relies on the relative areas of the signals above and below the mean.

Finally, we can suggest that other physiological control loops, all of which contribute to homeostasis and subject to blood flow/pressure pulsatility, could also potentially be examined by the equivalent nonlinearity principle. The main advantage of this method is that it gives analytical insight into the mechanisms at play and can lead to relatively simple nonlinear descriptions which can be further used in, for example, stability analysis.

\section{ACKNOWLEDGMENT}

The authors are grateful for the contribution to this work by Prof. Simon Malpas of the University of Auckland.

\section{REFERENCES}

[1] M. Chapleau, C. Heesch, and F. Abboud, "Prevention or attenuation of baroreceptor resetting by pulsatility during elevated pressure," Hypertension, vol. 9, no. III, pp. 137-141, 1987.

[2] F. Abboud and M. Chapleau, "Effects of pulse frequency on single-unit baroreceptor activity during sine-wave and natural pulses in dogs," $J$. Physiol, vol. 401, pp. 295-308, 1988.

[3] A. Guyton, T. Coleman, and H. Granger, "Circulation: overall regulation," Annu Rev Physiol., vol. 34, pp. 13-46, 1972.
[4] A. Monti, C. Mdigue, and M. Sorine, "Short-term modelling of the controlled cardiovascular system," ESIAM Proc., vol. 12, pp. 115-128, 2002.

[5] M. Ursino, "Interaction between carotid baroregulation and the pulsating heart: a mathematical model," A. J. Physiol. Heart Circ. Physiol., vol. 275, pp. H1733-H1747, 1998.

[6] R. Yozu, L. Golding, I. Yada, H. Harasaki, S. Takatani, S. Kawada, and Y. Nos, "Do we really need pulse? chronic nonpulsatile and pulsatile blood flow: From the exercise response viewpoints," Artificial Organs, vol. 18, pp. 638-642, 1994.

[7] A. Baba, P. Dobsak, S. Mochizuki, I. Saito, T. Isoyama, K. Takiura, M. Shibata, Y. Abe, T. Chinzei, J. Vasku, and K. Imachi, "Evaluation of pulsatile and nonpulsatile flow in microvessels of the bulbar conjunctiva in the goat with an undulation pump artificial heart," Artificial Organs, vol. 27, pp. 875-881, 2003.

[8] T. Drews, M. Jurmann, D. Michael, P. Miralem, Y. Weng, and R. Hetzer, "Differences in pulsatile and non-pulsatile mechanical circulatory support in long-term use," The Journal of Heart and Lung Transplantation, vol. 27, pp. 1096 - 1101, 2008.

[9] G. Preiss and C. Polosa, "Patterns of sympathetic neuron activity associated with mayer waves," American Journal of Physiology, vol. 226, pp. 724-730, 1974.

[10] R. L. Cooley, N. Montano, C. Cogliati, P. van de Borne, W. Richenbacher, R. Oren, and V. K. Somers, "Evidence for a central origin of the low-frequency oscillation in rr-interval variability," vol. 98, pp. 556-561, 1998.

[11] J. Ringwood and S. Malpas, "Control of renal blood flow - the case for a nonlinear model," Am. J. Physiol., vol. 280, pp. R1105-R1115, 2001

[12] A. de Paor and J. Ringwood, "A simple soft limiter describing function for biomedical applications," IEEE Trans. on Biomed. Eng., vol. 53, pp. $1233-1240,2006$

[13] S. Malpas, "Neural influences on cardiovascular variability: possibilities and pitfalls," Am. J. Physiol., vol. 282, pp. H6-H20, 2002.

[14] A. Avolio, M. O'Rourke, K. Mang, P. Bason, and B. Gow, “A comparative study study of pulsatile arterial haemodynamics in rabbits and guinea pigs," Am. J. Physiol, vol. 230, pp. 868-875, 1976.

[15] J. Lozier, "Carrier-controlled relay servos," Electrical engineering, vol. 69, pp. 1052-1056, 1950.

[16] R. Simpson and H. Power, "Applications of high frequency signal injection in non-linear systems," Int. J. Control, vol. 26, pp. 917-943, 1977.

[17] D. Atherton, Nonlinear control engineering. Van Nostrand Reinhold.

[18] W. Nichols and M. O'Rourke, McDonald's blood flow in arteries, 4th ed. Arnold, London, 1998. 